\title{
EFFECT OF SHADOWING AND MULTIPATH FADING ON THE AREA SPECTRAL FOR CELL-EDGE USERS IN HETEROGENEOUS NETWORKS
}

\author{
M. C. Uko1, M. A. Umoren ${ }^{2,}$ and J. Enyenihi ${ }^{3}$

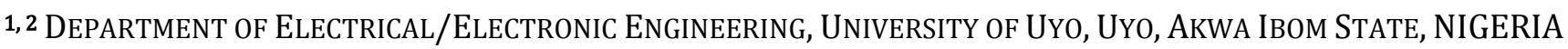 \\ 3 Dept. of Electrical/Electronic EnG' G, AKWA-Ibom State Univ., IKot AKPaden, AKWa Ibom State, NiGERIA \\ E-mail addresses:1mfonuko12@gmail.com,2mfon4gigis@yahoo.com,3gentlejay@hotmail.co.uk
}

\begin{abstract}
$A$ vital goal for communication operators is the need to provide communication resource to available consumers irrespective of their location. The traditional macro-only network have been proved ineffective when communication signal is required for users located in the cell edge and a certain distance away from the macrocell base station. This is because of excess attenuation of the signal due to fading and shadowing. Multipath fading and shadowing are major causes of performance degradation in mobile communication systems. This phenomenon which arises as a result of propagation effects (like scattering, diffraction and reflection), renders the traditional macro-only network communication with users far away from the macrocell base station and located in the cell edge less effective. The deployment of femtocells around the cell edge of this macrocell helps to reduce the effect of fading and shadowing thereby increasing the overall efficiency of the cellular network. Numerical simulation carried out for the macro-femto heterogeneous network using MATLAB shows how the effect of fading and shadowing was reduced. This holds a great promise for adaptive space-based wireless sensor networks, formationflying satellites and constellations.
\end{abstract}

Keywords: Femtocells, Heterogeneous networks, Macrocells, Spectral Efficiency, Uplink

\section{INTRODUCTION}

The presence of multiple paths is due to atmospheric scattering and refraction, or reflections from buildings and other objects [1]. This effect leads to degradation in the available communication resource for the data users. With the rapid evolving of the Wireless Industry, with vast developed and deployed infrastructure for providing many communication services for the data hungry users [2], user activities such as Voice over IP (VoIP), ultra-broadband access, real-time and streamed multimedia, gaming services and many other such activities has increased and hence requires increased bandwidth for flawless communication with high spectral efficiency [3]. The spectral efficiency, as an important measure of the performance of a communication system that deals with the effective transmission of data, must be properly maximized as a result of scarcity and high cost of available radio spectrum.

This efficiency must also be maximized at any given location of mobile users for a seamless communication. Any new communication strategy must be designed to use the available spectrum in the most efficient way in addition to providing optimum communication resources for the ever increasing users [4]. As mobile users increase, the pressure on the available communication spectrum also increases. This is as a result of huge data traffic caused by high data rate device users mostly situated within the mobile station, leaving users in the cell-edge with extremely poor reception of the communication resources from the base station.

To address these challenging necessities by way of improving coverage, capacity and deployment costs, heterogeneous network transmission techniques [57] are regarded to be one of the most promising solutions. A crucial part of these techniques will be how to significantly improve the capacity of users in the cell edge through the deployment of low-power and low-cost Femtocells [3] within and around the main macro cellular infrastructure. This is referred to as two-tier Heterogeneous network [8-10]. 
This paper considers the effect of multi-path fading and shadowing on the Area Spectral Efficiency (ASE) of this two-tier heterogeneous network in uplink called the Macro-Femto Feterogeneous Network (MFHN). The remaining part of this paper is structured as follows: Section 2 gives the overview of heterogeneous network; Section 3 describes the system model; Section 4 presents the simulation and discusses the results; Section 5 concludes.

\section{OVERVIEW OF HETEROGENEOUS NETWORK (HN)}

\subsection{Cellular Network Overview}

Fig. 1 shows a simplified diagram of a wireless mobile network transmitting with a macro base station. The signal is sampled and digitized after which it is encoded in binary form for modulation. The mobile transmitter transmits the modulated signal to the receiver of the base station in the uplink. The base station further determines the destination of the signal and then amplifies the signal for transmission to the receiving mobile device in the downlink. The mobile receiver receives the signal, demodulates it to its required form to ensure the decoding of the binary message. The decoded message is then forwarded to the output which could be the speaker of the mobile unit.

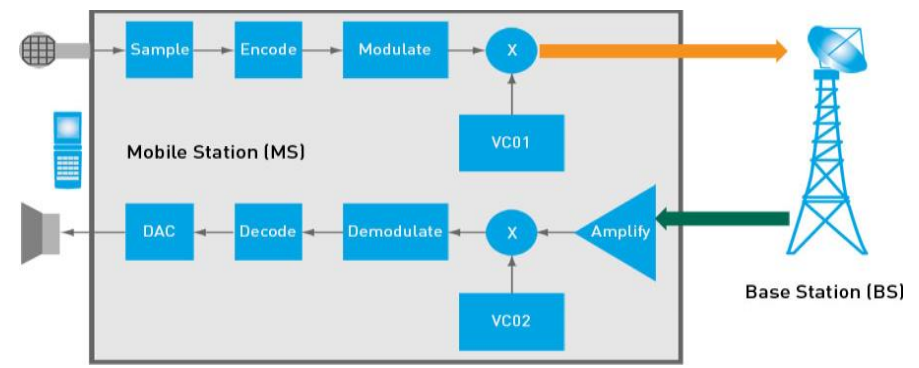

Fig. 1. A simplified cellular network transmission [11]

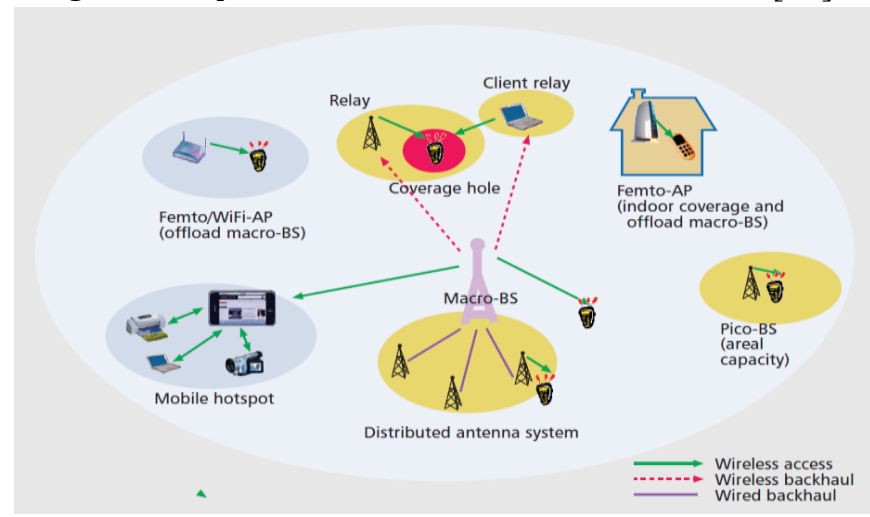

Fig 2. HN utilizing a combination of macro, pico, femto and relay base stations [15]

\subsection{Heterogeneous Network Concept}

Heterogeneous network is a network that consists of a combination of macrocells and low-power small cells e.g. Femtocells, Picocells, Distributed Antenna, Relay Node (RN) and Remote Radio Head (RRH). A cellular system that contains both macrocells and femtocell is referred to as a two-tier heterogeneous network (macro-femto) system. The small cell deployment shares the traffic load of macrocells whilst alleviating the capital and operational expenditures (CAPEX and OPEX) of macrocell base Stations [6, 14]. Moreover, the mobile users located within a given small cell enjoys high quality transmission due to their short distance from the serving Base Station, hence ensuring enhanced network capacity.

A breakdown of a typical heterogeneous network mix as shown in Fig. 2 is given below:

- Macrocells: These are generally designed to provide wide coverage and are made up of a high power base station. They are usually very high in height and costly to install.

- A Micro/Pico cell is a cell in a mobile phone network served by a low power cellular Base Station that covers a small area with dense traffic such as residential areas, universities, shopping mall, a hotel, or a train station. It has a coverage range of few hundred metres. Usually about three microcells can cover the same range of a singular macrocell.

- Femtocells are smaller cells spanning only a few metres. They are generally connected to a private owners' cable broadband connection or a home digital subscriber line (DSL). Femtocells because of their size are much more power efficient in providing wider coverage. An advantage of femtocells is their ability to use higher frequency bands suitable to provide high data rates. Femtocells also offer a cheap installation cost option as users can install them without a service provider.

However, deploying too many smaller cells around the cell edge of the macrocell may reduce the overall area spectral efficiency of the macrocell Base Station, causing the macrocell base station to operate under low load conditions. A careful analysis of various arrangement schemes should be done in order to find how to best deploy such smaller cells.

\section{SYSTEM MODEL AND ANALYSIS}

From Fig. 3, the first tier of the case study 
heterogeneous network comprises the macro-only network in which the carrier frequency is re-used at a minimum distance $D[\mathrm{~m}]$. This first tier also comprises of a circular macrocell of radius $R_{m}[\mathrm{~m}]$ with a base station made up of an omni-directional antenna. The user is considered to be randomly located within the macro-cell bounded by $R_{o}$ and $R_{m}$ where $R_{o}$ is the minimum distance a user can be with reference to the macrocell base station.

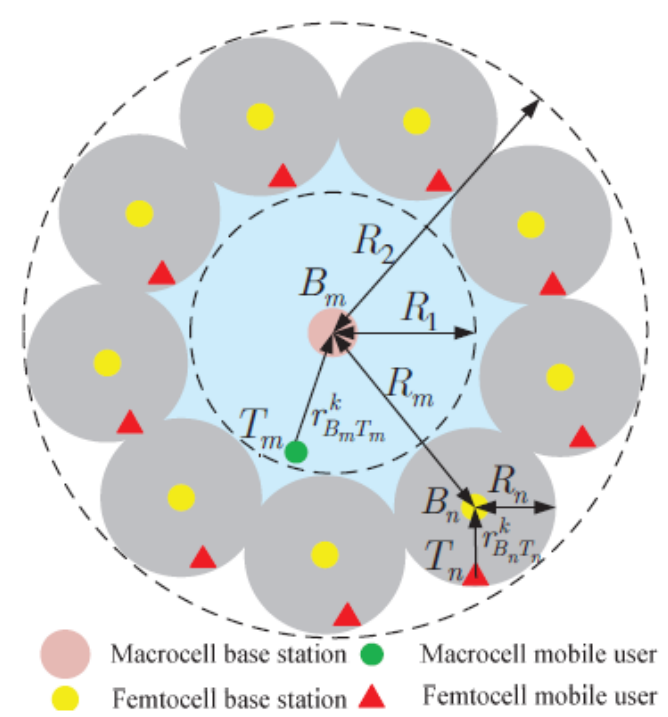

Fig. 3: Femtocells distribution at the cell edge in the Macro-Femto network. [16]

The second tier heterogeneous network is made up of $N$ circular femtocells each of radius $R_{n}[\mathrm{~m}]$ with lowpowered low-cost user deployed femto base station at the center. The femtocells are deployed round the edge of the reference macrocell. This is also referred to as the Femto-On-Edge (FOE) configuration in [16]. For the simulation, the number of femtocells per macrocell, $N$, is given as:

$$
N=\mu \frac{A_{m}}{A_{n}}
$$

where $A_{m}$ is the area of the macrocell, $A_{n}$ is the area of each of the femtocells and $\mu$ is referred to as the femto population factor (FPF) which controls the number of femtocells per macrocell.

\subsection{Spectral Efficiency of Wireless Networks}

Spectrum efficiency is the enhanced use of limited available spectrum or bandwidth so that the maximum amount of data can be transmitted with minimal transmission errors. In mobile communication, the spectrum efficiency is the maximum number of users per cell that can be accommodated while sustaining an acceptable quality of service $[16,17]$.

In [18], a performance based analysis of spectral efficiency of macro cellular system is introduced which is known as the area spectral efficiency defined as sum of mean achievable rates per unit bandwidth per unit area. Mathematically,

$$
A S E=\frac{\sum_{k=1}^{N_{s}} C_{k}}{\pi W(D / 2)^{2}}
$$

Where $N_{s}$ is the total number of active serviced channels per cell, $C_{k}[\mathrm{~b} / \mathrm{s}]$ is the maximum data rate of the kth user, $D$ is the frequency re-use distance and $W$ $[\mathrm{Hz}]$ which forms a critical part of this research is the assigned bandwidth per cell.

\subsection{Bandwidth Allocation}

In this paper, co-channel allocation of the bandwidth is used. Here, the users all share the same frequency channel without any partitioning. This is to say that:

$$
W_{m}=W_{f}=W
$$

where $W_{m}$ is the bandwidth for the macrocell users, $W_{f}$ is the bandwidth for the femtocell users and $W$ is the total available bandwidth. The macrocell and the femtocells share all the communication resources available. For simplicity, the channel is assumed to be serving only one user at a time for the both tier. The bandwidth is re-used throughout the macrocell network at a distance $D=R_{u}\left(R_{m}+R_{n}\right)$ where $R_{u}$ is the network traffic load with a value of 2 for a fully loaded cellular network.

\subsection{Mobile User Distribution}

The mobile users in the macrocell, femtocell and interfering cells are assumed to be independent and uniformly distributed in their cells. The joint probability density function (PDF) of the macrocell users at any location $(r, \theta)$ from its serving macrocell base station is given as

$$
P(r, \theta)=\frac{r-R_{o}}{\pi\left(R_{1}-R_{o}\right)^{2}}, R_{\theta}(\theta)=\frac{1}{2 \pi}
$$

Where $R_{0} \leq r \leq R_{1}, 0 \leq \theta \leq 2 \pi$ and $R_{1}=R_{m}-R_{n}$ For the femtocell users at any location $(\tilde{r}, \theta)$ from its serving femtocell base station, the PDF is given as:

$$
P(\bar{r}, \theta)=\frac{\bar{r}}{\pi R_{n}{ }^{2}}, R_{\bar{\theta}}(\bar{\theta})=\frac{1}{2 \pi}
$$

where $0 \leq \bar{r} \leq R_{n}$, and $0 \leq \bar{\theta} \leq 2 \pi$. 


\subsection{Shadowing}

This models the signal power at same T-R separation but at different locations. Shadowing is caused by obstacles like building that affect signal power through reflection, scattering, and diffraction. This effect also varies geographically. The modelling of this effect on communication systems is usually random as desired mobile user's experiences this effect randomly due to motion or change in environment. The shadowing is modelled as a lognormal distribution with the probability density function (PDF) of the slowly varying received signal power given as:

$$
P s(P)=\frac{\xi}{\sqrt{2 \pi} \sigma P} \exp \left(-\frac{(\xi \ln (P)-\mu)^{2}}{2 \sigma^{2}}\right), P \geq 0
$$

where $\xi=10 / \ln 10, \mu=\xi \operatorname{in}(\bar{P})$ is the logarithmic mean power in $\mathrm{dB}, \sigma$ is the shadow standard deviation in $\mathrm{dB}$.

\subsection{Fading}

Mobile channel is characterized by two types of fading effect:

\section{i. Large-scale fading \\ ii. Small scale fading}

Large-scale fading is the slow deviation of the mean signal power over time. This is basically dependent on the existence of obstacle in the signal path and on the relative position of the mobile user. This slow deviation is modelled as having lognormal distribution. Small -scale fading is basically of two types: Rayleigh or Rician fading which are dependent on the number of reflective paths encountered. Rayleigh fading occurs if the reflective paths are large in number and there is no principal line-of-sight (LOS) propagation path. Rician fading occurs when there is a principal LOS path. This is modelled using the slow varying flat fading channel. It is assumed that the fading environment is characterized by a Nakagami-m distribution with the probability density function (PDF) of the received signal power given as:

$$
P s(P)=\left(\frac{m}{\Omega}\right)^{m} \frac{P^{m-1}}{\Gamma(m)} \exp \left(-\mathrm{m} \frac{\mathrm{P}}{\Omega}\right), P \geq 0
$$

where $m$ is the Nakagami fading parameter, $\Omega$ is the mean received power related to path-loss and shadowing, $\Gamma($.$) is the gamma function.$

\subsection{Area Spectral Efficiency}

The Area spectral efficiency is defined as the sum of the maximum available rates per bandwidth per unit macro-cell area. For the two tier network being considered, mathematically the ASE can be expressed as $[16,17]$ :

$$
A S E_{\text {macro }+ \text { femto }}=\frac{4\left(W_{m} C_{m}+N W_{f} C_{f}\right)}{\pi W R_{u}^{2}\left(R_{m}+R_{n}\right)^{2}}
$$

$W_{m}$ is the bandwidth of the macrocell, $W_{f}$ is the bandwidth of the femtocell, $C_{m}$ is the spectral efficiency (Capacity) of the macrocell, $C_{f}$ is the capacity of the femtocell and $\mathrm{N}$ is the number of femtocell deployed.

From the earlier assumption in equation (1) this equation reduces to

$$
A S E_{\text {macro }+ \text { femto }}=\frac{4\left(C_{m}+N C_{f}\right)}{\pi R_{u}^{2}\left(R_{m}+R_{n}\right)^{2}}
$$

\section{SIMULATION AND RESULTS}

A Monte-Carlo simulation procedure is established for the given system parameters.

Table 1: Simulation parameters values

\begin{tabular}{l|c|c}
\hline Simulation parameters & Femtocell & Macrocell \\
\hline System bandwidth & \multicolumn{2}{|c}{$20 \mathrm{MHz}$} \\
\hline Cell radius & $30 \mathrm{~m}$ & $100-600 \mathrm{~m}$ \\
\hline Path-Loss Exponent & 2 & 2 \\
\hline Additional path-loss exponent & 2 & 2 \\
\hline BS antenna height & $5 \mathrm{~m}$ & $25 \mathrm{~m}$ \\
\hline Mobile User antenna height & $1.5 \mathrm{~m}$ & $1.5 \mathrm{~m}$ \\
\hline Femto Population Factor, $\mu$ & \multicolumn{2}{|c}{1} \\
\hline Reference distance & \multicolumn{2}{|c}{100} \\
\hline Path-loss constant, $\mathrm{K}$ & \multicolumn{2}{|c}{1} \\
\hline Maximum transmit Power & \multicolumn{2}{|c}{$10 \mathrm{Watt}$} \\
\hline Reference distance, $\mathrm{R}_{\mathrm{o}}$ & - & 100 \\
\hline \hline
\end{tabular}

\subsection{Area Spectral Efficiency with Shadowing}

In this section, the effect of shadowing is investigated. Shadowing occurs due to objects obstructing the relative propagation path between the transmitter and receiver. For long distance propagation, the received signal is modelled as a log-normal distribution with values in $\mathrm{dB}$. A case of light shadowing $\left(\sigma_{I}=4 \mathrm{~dB}\right)$ and heavy shadowing $\left(\sigma_{I}=6\right.$ $\mathrm{dB})$ are considered.

Fig 4 shows the effect on the area spectral efficiency for a shadowing parameter of $4 \mathrm{~dB}$. From Fig. 4, a lognormal shadowing parameter of $4 \mathrm{~dB}$ reduces the ASE for the macro only. For the macro-femto network, shadowing effect is negligible. In Fig. 5, an increase in the shadowing parameter to $6 \mathrm{~dB}$ further reduces the area spectral efficiency of the macro-only network. The area spectral efficiency of the macro-femto network is minimally affected. 


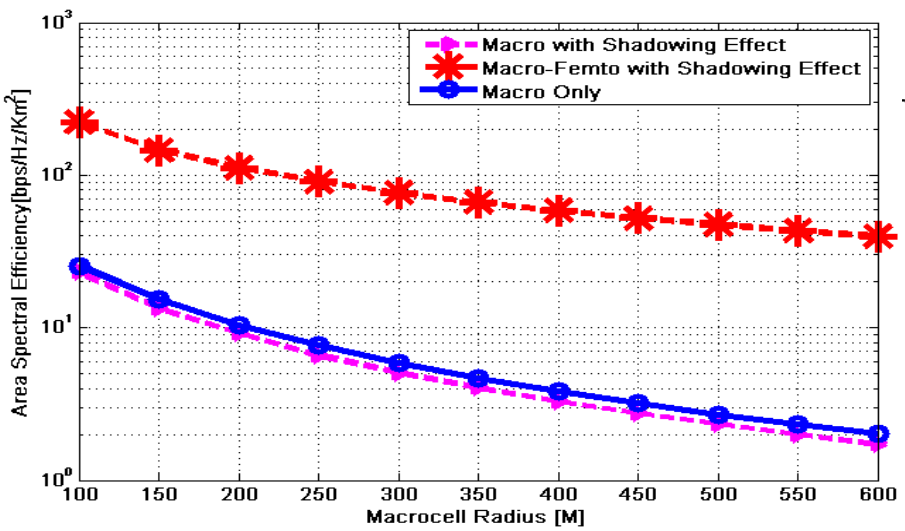

Fig. 4. Effect of shadowing on the area spectral efficiency for

$$
\sigma_{I}=4 d B
$$

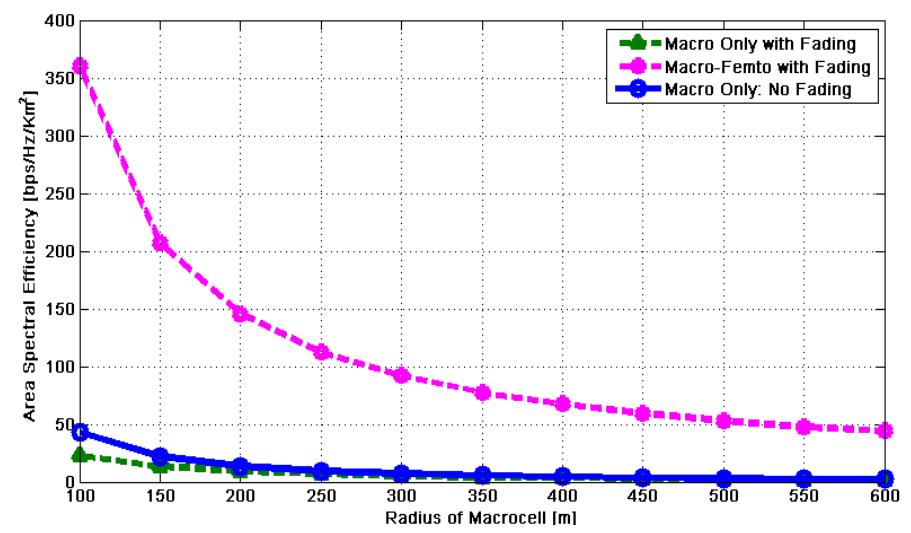

Fig. 6. Effect of fading on the area spectral efficiency for $m_{I}$

$$
=1
$$

Comparing Figs. 4 and 5, the lower effect of shadowing on the macro-femto network can be attributed to the deployment of the low powered femtocells at the cell edge which provides the platform of signal reception for the cell-edge user rather than receiving communication signal directly from the traditional macro base station which is subject to more shadowing effect.

Furthermore, the deployment of the femtocells provides the medium for cell-edge users' connection to the network thus reducing the traffic on the traditional microcell network. This leads to less shadowing experienced over the macro-only distance, hence improving the quality of the over-all macrofemto network.

\subsection{Area Spectral Efficiency with Fading}

Fading is the distortion to communication signal as it is being propagated through certain propagation medium. This distortion may be as a result of multiple reflection of transmitted signal from various surfaces leading to a multipath propagation of the transmitted

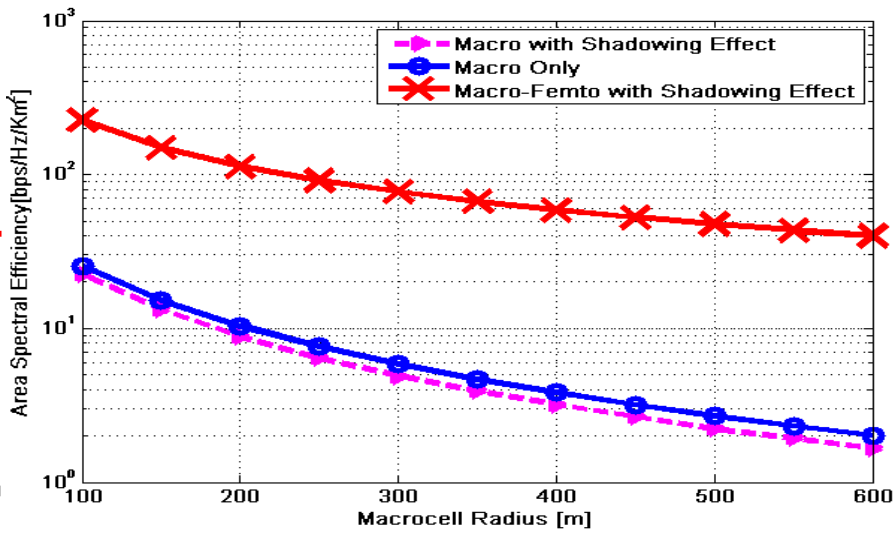

Fig. 5. Effect of shadowing on the area spectral efficiency for

$$
\sigma_{I}=6 d B
$$

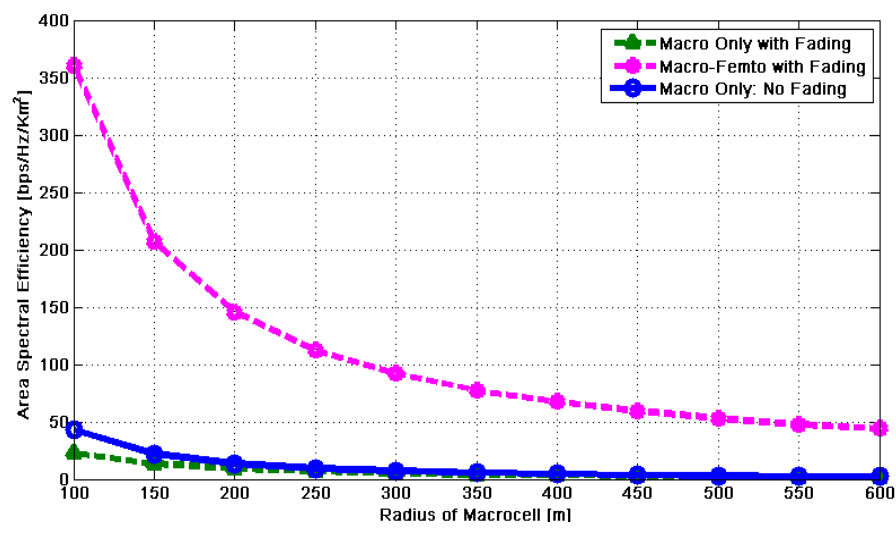

Fig.7. Effect of fading on the area spectral efficiency for $m_{I}$

$$
=3
$$

signal. This effect is considered for mobile users situated in the cell edge where they are prone to excessive fading on the communication signal from the traditional macrocell base station.

From Fig.6 and Fig. 7, as the interfering mobile user increases from $m_{I}=1$ to $m_{I}=3$, the degradation on a Macro-only network reduces the spectral efficiency when compared with the Macro-Femto Heterogeneous network. There is negligible effect of the fading on the Macro-Femto Heterogeneous network. In a deep fade scenario, the Macro-Femto network performs better than the Macro-only network as the radius of the macrocell is increased.

\section{CONCLUSION}

In this paper, a two-tier heterogeneous network in uplink called Macro-Femto Heterogeneous Network (MFHN) has been proposed. The system model of the Macro-Femto network is presented and analyzed, and its comparative advantage discussed. Using MATLAB and a Monte-Carlo simulation process, the effect of fading and shadowing on the area spectral efficiency 
of the macro-femto network (MFHN) was investigated. A comparative analysis was also carried out with a macro-only network. Using shadowing as metric, it was observed from the simulation results that, the effect of shadowing on the area spectral efficiency of the macro-femto network was negligible compared to macro-only network (with or without shadowing). Using fading as metric, it was also observed from the simulation result that, the effect of fading on the area spectral efficiency of the macrofemto network was negligible compared to macroonly network (with or without shadowing). Finally, this paper has established that with increase in the interfering mobile user, shadowing and fading reduces the spectral efficiency on a Macro-only network while their effect on the Macro-Femto Heterogeneous network is negligible.

\section{REFERENCES}

[1] Michel C. Jeruchim, Philip Balaban and K. Sam Shanmugan. "Communication Systems Modeling, Mathodology, and Techniques", Kluwer Academic Publishers. New York.

[2] M.S. Chavan, R. H. Chile and S.R. Sawant. "Multipath Fading Channel Modeling and Performance Comparison of Wireless Channel Models. International Journal of Electronics and Communication Engineering", Volume 4, Number 2 (2011), Pp. 189-203.

[3] Bhargavi D. K. And Dr. Vijaya Prakash A. M, "A Novel Handover Algorithm for LTE Based MacroFemto Heterogeneous Networks" International Journal of VLSI Design \& Communication Systems (VLSICS) Vol.6, No.4, August 2015

[4] Syed A. Jafar, Gerard J. Foschini and Andrea J. Goldsmith "Phantomnet: Exploring Optimal Multicellular Multiple Antenna Systems", EURASIP Journal on Applied Signal Processing 2004:5, 591604

[5] R. Pabst, "Relay-Based Deployment Concepts for Wireless and Mobile Broadband Radio," IEEE Communication Magazine, Vol. 42, Pp. 80-89, Sept. 2004.

[6] D. Soldani and S. Dixit, "Wireless Relays For Broadband Access," IEEE Commun. Mag., Vol. 46, Pp. 58-66, Mar. 2008.

[7] A. Sendonaris, E. Erkip, and B. Aazhang, "User Cooperation Diversity. Part I and II," IEEE Trans. Commun., Vol. 51, Pp. 1927-1948, Nov. 2003.
[8] P. Lin, J. Zhang, Y. Chen, and Q. Zhang, "MacroFemto Heterogeneous Network Deployment and Management: From Business Models to Technical Solutions," IEEE Wireless Communs. Mag., Vol. 18, No. 3, Pp.64-70, Jun. 2011.

[9] S. Landstrom, A. Furuskar, K. Johansson, L. Falconetti, And F. Kronestedt, "Heterogeneous Networks Increasing Cellular Capacity," Jour. Ericson Review, Vol. 89, Pp. 4-9, Jan. 2011.

[10] Femto Forum, "An Overview of the Femtocell Concept." [Online]. Available: Www.Femtoforum.Org.

[11] A. L. Stolyar and H. Viswanathan, "Self-Organizing Dynamic Fractional Frequency Reuse for BestEffort Traffic through Distributed Inter-Cell Coordination," In Proc. IEEE INFOCOM, Rio De Janeiro, Brazil, Apr. 2009, Pp. 1-9.

[12] V. Chandrasekhar, J. G. Andrews, and A. Gatherer, "Femtocell Networks: A Survey," IEEE Commun. Mag., Vol. 46, No. 9, Pp. 59-67, Sept. 2008.

[13] W. H. Tranter, K. S. Shanmugan, T. S. Rappaport, "Principles Of Communication Systems Simulation With Wireless Applications" Prentice Hall, 2003, Pp. 1-760.

[14]

Http://Www.Symmetricom.Com/Resources/Ne wsletters/Just-A-Second-Archives/November2009/IMPACT-OF-PHASE-NOISE-ON-CELLPHONES

[15] Shu-Ping Yeh, "Capacity and Coverage Enhancement In Heterogeneous Networks," IEEE European Wireless Conference, Vol. 18, Issue: 3, June 2011, Pp. 32- 38.

[16] M. Z. Shakir and M.-S. Alouini, "On The Area Spectral Efficiency Improvement Of Heterogeneous Network By Exploiting The Integration Of Macro-Femto Cellular Networks," In Proc. IEEE Intl. Conf. Communs., (ICC'12), Pp. 1-6, Ottawa, Canada, Jun. 2012.

[17] Uko, M. C.; Ekpo, S. C.; Ukommi, U.; Kharel, R. "Shadowing Effect On Macro-Femto Heterogeneous Network For Cell-Edge Users" Applied Computational Electromagnetics (ACES), 2015 31st International Review Of Progress In Applied Computational Electromagnetics year: 2015 Pages: 1 - 2

[18] P. S. Hall, Z. H. Hu, and Y. I. Nechayev, "Short Term and Long Term Fading of On -Body Transmission Channels At 2.45ghz," Antennas 7 Propagation Conference Loughborough (LAPC, 2009), 16-17 Nov. 2009, Pp. 657-660. 